Commun. Korean Math. Soc. 29 (2014), No. 2, pp. 239-255

http://dx.doi.org/10.4134/CKMS.2014.29.2.239

\title{
SHIFTED HARMONIC SUMS OF ORDER TWO
}

\author{
AnTHONY SOFO
}

Abstract. We develop a set of identities for Euler type sums. In particular we investigate products of shifted harmonic numbers of order two and reciprocal binomial coefficients.

\section{Introduction and preliminaries}

In this paper we study the summation of the product shifted harmonic sums of order two and reciprocal binomial coefficients, of the form,

$$
\sum_{n=1}^{\infty} \frac{H_{n-\frac{1}{q}}^{(2)}}{n\left(\begin{array}{c}
n+k \\
k
\end{array}\right)}
$$

and its finite counterpart, in the process we shall obtain some new two parameter hypergeometric function identities. Analogous results of Euler type for infinite series have been developed by many authors, see for example [2], and references therein. Let,

$$
H_{n}=\gamma+\psi(n+1)=\sum_{j \geq 1} \frac{n}{j(j+n)}=\sum_{r=1}^{n} \frac{1}{r}=\int_{0}^{\infty} \frac{1-t^{n}}{1-t} d t
$$

be the $n^{\text {th }}$ harmonic number, $\gamma$ denotes the Euler-Mascheroni constant,

$$
\psi(z):=d \log \Gamma(z) / d z
$$

is the digamma function and $\Gamma(z)$ is the well known gamma function.

Let $\mathbb{R}$ and $\mathbb{C}$ denote, respectively the sets of real and complex numbers and $\mathbb{N}:=\{1,2,3, \ldots\}$ the set of natural numbers. A generalized binomial coefficient $\left(\begin{array}{c}w \\ z\end{array}\right)$ may be defined by

$$
\left(\begin{array}{l}
w \\
z
\end{array}\right):=\frac{\Gamma(w+1)}{\Gamma(z+1) \Gamma(w-z+1)} ; w, z \in \mathbb{C}
$$

Received September 30, 2013.

2010 Mathematics Subject Classification. Primary 05A10, 05A19, 11B65; Secondary 11B83, 11M06, 33B15, 33D60, 33C20.

Key words and phrases. harmonic numbers, binomial coefficients and gamma function, polygamma function, combinatorial series identities and summation formulas, partial fraction approach, hypergeometric identity. 
and in the special case when $z=n, n \in \mathbb{N}$, we have

$$
\left(\begin{array}{l}
w \\
n
\end{array}\right):=\frac{w(w-1) \cdots(w-n+1)}{n !}=\frac{(-1)^{n}(-w)_{n}}{n !}
$$

where

$$
\begin{aligned}
(w)_{\lambda} & :=\frac{\Gamma(w+\lambda)}{\Gamma(w)} \\
& =\left\{\begin{array}{l}
1, \lambda=0 ; w \in \mathbb{C} \backslash\{0\} \\
w(w+1) \cdots(w+\lambda-1), w \in \mathbb{C}, \lambda \in \mathbb{N},
\end{array} \quad \text { with }(0)_{0}:=1\right.
\end{aligned}
$$

is known as the Pochhammer symbol.

Some well known Euler sums are, [4],

$$
\sum_{n=1}^{\infty} \frac{H_{n}^{3}-3 H_{n} H_{n}^{(2)}+2 H_{n}^{(3)}}{(n+1)^{2}}=6 \zeta(5),
$$

in [14] we have, for $k \geq 1$

$$
\sum_{n=1}^{\infty} \frac{H_{n}^{(2)}}{n\left(\begin{array}{c}
n+k \\
k
\end{array}\right)}=\zeta(3)-H_{k-1} \zeta(2)+\sum_{r=1}^{k}(-1)^{r}\left(\begin{array}{c}
k \\
r
\end{array}\right) \sum_{j=1}^{r-1} \frac{H_{j}}{j^{2}}
$$

and [18],

$$
\begin{aligned}
& \sum_{n=1}^{p} \frac{H_{n}^{(2)}}{n\left(\begin{array}{c}
n+k \\
k
\end{array}\right)} \\
& =H_{p}^{(3)}-H_{p}^{(2)}\left(H_{k-1}+\frac{1}{k\left(\begin{array}{c}
k+p \\
k
\end{array}\right)}\right) \\
& +\frac{H_{p}}{2}\left(H_{k}^{(2)}+H_{k}^{2}-\frac{2 H_{k}}{k}\right)-\sum_{r=1}^{k}(-1)^{r}\left(\begin{array}{c}
k \\
r
\end{array}\right) \sum_{j=1}^{r-1} \frac{H_{j+p}-H_{j}}{j^{2}},
\end{aligned}
$$

where the generalized $n^{\text {th }}$ harmonic number in power $r, H_{n}^{(r)}$, is defined for positive integers $n$ and $r$ as

$$
H_{n}^{(r)}:=\sum_{m=1}^{n} \frac{1}{m^{r}}
$$

Many finite versions of harmonic number sum identities also exist in the literature, for example in, [10] we have

$$
\sum_{k=0}^{2 m}(-1)^{k}\left(\begin{array}{c}
2 m \\
k
\end{array}\right)\left(H_{k}^{2}+H_{k}^{(2)}-H_{k} H_{2 m-k}\right)=\frac{(-1)^{m}}{4}\left(\begin{array}{c}
2 m \\
m
\end{array}\right) H_{m}^{(2)},
$$


and from, $[8]$

$$
\sum_{k=0}^{n}(-1)^{n} H_{k} H_{k}^{(2)}=(n+1) H_{n} H_{n}^{(2)}-\frac{2 n+1}{2} H_{n}^{(2)}+H_{n}-\frac{1}{2} H_{n}^{2} .
$$

Further work in the summation of harmonic numbers and binomial coefficients has also been done by Sofo [16]. The works of, [1], [5], [6], [7], [8], [10], [11], $[15],[20],[12],[13],[17],[19]$, and references therein, also investigate various representations of binomial sums and zeta functions in simpler form by the use of the Beta function and by means of certain summation theorems for hypergeometric series.

Lemma 1. Let $q \neq 0, m, p$ and $r$ be positive integers. Then

$$
\begin{aligned}
\sum_{j=1}^{p} \frac{q^{2}}{(m+j)(q j-1)^{2}}= & \frac{q^{2}}{(q m+1)^{2}}\left(H_{p+m}-H_{m}-H_{p-\frac{1}{q}}+H_{-\frac{1}{q}}\right) \\
& +\frac{q}{q m+1}\left(H_{p-\frac{1}{q}}^{(2)}-H_{-\frac{1}{q}}^{(2)}\right), \\
\sum_{j=1}^{\infty} \frac{q}{(m+j)(q j-1)}= & \frac{q^{2}}{(q m+1)^{2}}\left(H_{-\frac{1}{q}}-H_{m}\right)-\frac{q}{q m+1} H_{-\frac{1}{q}}^{(2)} \\
\sum_{m=1}^{r-1} \frac{q^{2} H_{m}}{(q m+1)^{2}}= & H_{r-1} H_{r+\frac{1}{q}-1}^{(2)}-\sum_{s=1}^{r-1} \frac{H_{s+\frac{1}{q}-1}^{(2)}}{s},
\end{aligned}
$$

and

$$
\sum_{m=1}^{r-1}\left(\frac{q}{q m+1}\right)^{2}=H_{\frac{1}{q}}^{(2)}-H_{r+\frac{1}{q}-1}^{(2)}
$$

Proof. For (1.3),

$$
\begin{aligned}
& \sum_{j=1}^{p} \frac{q^{2}}{(m+j)(q j-1)^{2}} \\
= & \sum_{j=1}^{p} \frac{q^{2}}{(q m+1)^{2}(m+j)}+\sum_{j=1}^{p} \frac{q^{3}}{(q m+1)(q j-1)^{2}}-\sum_{j=1}^{p} \frac{q^{3}}{(q m+1)^{2}(q j-1)} \\
= & \frac{q^{2}}{(q m+1)^{2}}\left(H_{p+m}-H_{m}\right)+\frac{q^{2}}{(q m+1)^{2}}\left(H_{-\frac{1}{q}}-H_{p-\frac{1}{q}}\right) \\
& +\frac{q}{q m+1}\left(H_{p-\frac{1}{q}}^{(2)}-H_{-\frac{1}{q}}^{(2)}\right) .
\end{aligned}
$$

Similarly for (1.4). For (1.5) we first note that for an arbitrary sequence $X_{k, l}$ the following identity holds

$$
\sum_{k=1}^{n} \sum_{l=1}^{k} X_{k, l}=\sum_{l=1}^{n} \sum_{k=l}^{n} X_{k, l}
$$


hence

$$
\begin{aligned}
\sum_{m=1}^{r-1} \frac{q^{2} H_{m}}{(q m+1)^{2}} & =\sum_{m=1}^{r-1} \sum_{s=1}^{m} \frac{q^{2}}{s(q m+1)^{2}}=\sum_{s=1}^{r-1} \sum_{m=s}^{r-1} \frac{q^{2}}{s(q m+1)^{2}} \\
& =\sum_{s=1}^{r-1} \frac{1}{s}\left(H_{r+\frac{1}{q}-1}^{(2)}-H_{s+\frac{1}{q}-1}^{(2)}\right) \\
& =H_{r-1} H_{r+\frac{1}{q}-1}^{(2)}-\sum_{s=1}^{r-1} \frac{H_{s+\frac{1}{q}-1}^{(2)}}{s} .
\end{aligned}
$$

For (1.6)

$$
\sum_{m=1}^{r-1}\left(\frac{q}{q m+1}\right)^{2}=\sum_{m=1}^{r-1} \frac{1}{\left(m+\frac{1}{q}\right)^{2}}=\sum_{m=1+\frac{1}{q}}^{r+\frac{1}{q}-1} \frac{1}{m^{2}}=H_{\frac{1}{q}}^{(2)}-H_{r+\frac{1}{q}-1}^{(2)} .
$$

In the case of non integer values of the argument $z=\frac{r}{q}$, we may write the generalized harmonic numbers, $H_{z}^{(\alpha+1)}$, in terms of polygamma functions

$$
H_{\frac{r}{q}}^{(\alpha+1)}=\zeta(\alpha+1)+\frac{(-1)^{\alpha}}{\alpha !} \psi^{(\alpha)}\left(\frac{r}{q}+1\right), \frac{r}{q} \neq\{-1,-2,-3, \ldots\},
$$

where $\zeta(z)$ is the zeta function. When we encounter harmonic numbers at possible rational values of the argument, of the form $H_{\frac{r}{q}}^{(\alpha)}$ they maybe evaluated by an available relation in terms of the polygamma function $\psi^{(\alpha)}(z)$ or, for rational arguments $z=\frac{r}{q},(1.7)$ and we also define

$$
H_{\frac{r}{q}}^{(1)}=\gamma+\psi\left(\frac{r}{q}+1\right) \text {, and } H_{0}^{(\alpha)}=0 .
$$

The evaluation of the polygamma function $\psi^{(\alpha)}\left(\frac{r}{a}\right)$ at rational values of the argument can be explicitly done via a formula as given by Kölbig [9], or Choi and Cvijovic [3] in terms of the Polylogarithmic or other special functions. Some specific values are given as

$$
\begin{aligned}
& H_{\frac{3}{4}}^{(3)}=\frac{64}{27}+\pi^{3}-27 \zeta(3), \quad H_{\frac{3}{2}}^{(1)}=\frac{8}{3}-2 \ln (2), \\
& H_{\frac{3}{4}}^{(1)}=\frac{4}{3}+\frac{\pi}{2}-3 \ln (2), \text { and } H_{\frac{7}{4}}^{(2)}=\frac{928}{441}+8 G-5 \zeta(2) .
\end{aligned}
$$

many others are listed in the excellent book [21].

\section{Harmonic number identities}

We now prove the following two theorems. 
Theorem 1. Let $k \in \mathbb{N}$ and for real number $q>1$ and $q<-1$. Then we have

$$
\begin{aligned}
& \sum_{n=1}^{\infty} \frac{H_{n-\frac{1}{q}}^{(2)}}{n\left(\begin{array}{c}
n+k \\
k
\end{array}\right)} \\
= & H_{-\frac{1}{q}}^{(2)}\left(\frac{1}{k}+H_{\frac{1}{q}-1}\right)-H_{-\frac{1}{q}} H_{\frac{1}{q}-1}^{(2)}-\zeta(2) H_{\frac{1}{q}-1}+\sum_{r=1}^{k}(-1)^{r+1}\left(\begin{array}{c}
k \\
r
\end{array}\right) \\
& \times\left[\left(\zeta(2)-H_{-\frac{1}{q}}^{(2)}\right) H_{r+\frac{1}{q}-1}+H_{r+\frac{1}{q}-1}^{(2)}\left(H_{-\frac{1}{q}}-H_{r-1}\right)+\sum_{s=1}^{r-1} \frac{H_{s+\frac{1}{q}-1}^{(2)}}{s}\right] .
\end{aligned}
$$

Proof. Let $h_{n}^{(2)}=H_{n-\frac{1}{q}}^{((2))}-H_{-\frac{1}{q}}^{(2)}$ and consider the following expansion:

$$
\sum_{n=1}^{\infty} \frac{h_{n}^{(2)}}{n\left(\begin{array}{c}
n+k \\
k
\end{array}\right)}=\sum_{n=1}^{\infty} \frac{k ! h_{n}^{(2)}}{n \prod_{r=1}^{k}(n+r)}=\sum_{n=1}^{\infty} \frac{k ! h_{n}^{(2)}}{n(n+1)_{k+1}}
$$

Now

$$
\sum_{n=1}^{\infty} \frac{h_{n}^{(2)}}{n\left(\begin{array}{c}
n+k \\
k
\end{array}\right)}=\sum_{n=1}^{\infty} \frac{k ! h_{n}^{(2)}}{n} \sum_{r=1}^{k}\left(\frac{A_{r}}{n+r}\right)
$$

where

$$
A_{r}=\lim _{n \rightarrow-r} \frac{n+r}{\prod_{r=1}^{k} n+r}=\frac{(-1)^{r+1} r}{k !}\left(\begin{array}{c}
k \\
r
\end{array}\right)
$$

For an arbitrary positive sequence $X_{k, p}$ the following identity holds

$$
\sum_{k=0}^{\infty} \sum_{p=0}^{n} X_{p, k}=\sum_{k=0}^{\infty} \sum_{p=0}^{\infty} X_{p, k+p}
$$

hence from (2.2)

$\sum_{n=1}^{\infty} \frac{k ! h_{n}^{(2)}}{n} \sum_{r=1}^{k}\left(\frac{A_{r}}{n+r}\right)=\sum_{r=1}^{k}(-1)^{r+1} r\left(\begin{array}{c}k \\ r\end{array}\right) \sum_{n=1}^{\infty} \frac{1}{n(n+r)} \sum_{j=1}^{n} \frac{q^{2}}{(q j-1)^{2}}$

where

$$
h_{n}^{(2)}=H_{n-\frac{1}{q}}^{((2))}-H_{-\frac{1}{q}}^{(2)}=\sum_{j=1}^{n} \frac{q^{2}}{(q j-1)^{2}} .
$$




$$
\begin{aligned}
\sum_{n=1}^{\infty} \frac{h_{n}^{(2)}}{n\left(\begin{array}{c}
n+k \\
k
\end{array}\right)} & =\sum_{r=1}^{k}(-1)^{r+1} r\left(\begin{array}{l}
k \\
r
\end{array}\right) \sum_{j=1}^{\infty} \frac{q^{2}}{(q j-1)^{2}} \sum_{n=0}^{\infty} \frac{1}{(n+j)(n+j+r)} \\
& =\sum_{r=1}^{k}(-1)^{r+1} r\left(\begin{array}{l}
k \\
r
\end{array}\right) \sum_{j=1}^{\infty} \frac{q^{2}}{(q j-1)^{2}}\left[\frac{\psi(j+r)-\psi(j)}{r}\right] .
\end{aligned}
$$

Since we notice that

$$
\psi(j+r)-\psi(j)=\sum_{m=0}^{r-1} \frac{1}{m+j}
$$

then

$$
\begin{aligned}
\sum_{n=1}^{\infty} \frac{h_{n}^{(2)}}{n\left(\begin{array}{c}
n+k \\
k
\end{array}\right)} & =\sum_{r=1}^{k}(-1)^{r+1}\left(\begin{array}{c}
k \\
r
\end{array}\right) \sum_{m=0}^{r-1} \sum_{j=1}^{\infty} \frac{q^{2}}{(q j-1)^{2}(m+j)} \\
& =\sum_{r=1}^{k}(-1)^{r+1}\left(\begin{array}{c}
k \\
r
\end{array}\right) q^{2} \sum_{m=0}^{r-1}\left[\frac{H_{-\frac{1}{q}}-H_{m}}{(q m+1)^{2}}+\frac{\zeta(2)-H_{-\frac{1}{q}}^{(2)}}{q(q m+1)}\right]
\end{aligned}
$$

From (1.4), (1.5) and (1.6)

$$
\begin{aligned}
& \sum_{n=1}^{\infty} \frac{h_{n}^{(2)}}{n\left(\begin{array}{c}
n+k \\
k
\end{array}\right)} \\
= & \sum_{r=1}^{k}(-1)^{r+1}\left(\begin{array}{c}
k \\
r
\end{array}\right)\left[\begin{array}{c}
H_{-\frac{1}{q}}\left(H_{r+\frac{1}{q}-1}^{(2)}-H_{\frac{1}{q}-1}^{(2)}\right)+q^{2} \sum_{m=1}^{r-1} \frac{H_{m}}{(q m+1)^{2}} \\
+\left(\zeta(2)-H_{-\frac{1}{q}}^{(2)}\right)\left(H_{r+\frac{1}{q}-1}-H_{\frac{1}{q}-1}\right)
\end{array}\right] \\
= & \sum_{r=1}^{k}(-1)^{r+1}\left(\begin{array}{c}
k \\
r
\end{array}\right)\left[\begin{array}{c}
H_{-\frac{1}{q}}\left(H_{r+\frac{1}{q}-1}^{(2)}-H_{\frac{1}{q}-1}^{(2)}\right)+\left(\zeta(2)-H_{-\frac{1}{q}}^{(2)}\right)\left(H_{r+\frac{1}{q}-1}-H_{\frac{1}{q}-1}\right) \\
-H_{r-1} H_{r+\frac{1}{q}-1}^{(2)}+\sum_{s=1}^{r-1} \frac{H_{s+\frac{1}{q}-1}^{(2)}}{s}
\end{array}\right] .
\end{aligned}
$$

Now

$$
\sum_{n=1}^{\infty} \frac{h_{n}^{(2)} n\left(\begin{array}{c}
n+k \\
k
\end{array}\right)}{k} \sum_{r=1}^{k}(-1)^{r+1}\left(\begin{array}{c}
k \\
r
\end{array}\right)\left[\begin{array}{c}
-H_{-\frac{1}{q}} H_{\frac{1}{q}-1}^{(2)}-H_{-\frac{1}{q}-1}\left(\zeta(2)-H_{-\frac{1}{q}}^{(2)}\right) \\
\left(\zeta(2)-H_{-\frac{1}{q}}^{(2)}\right) H_{r+\frac{1}{q}-1}-H_{r-1} H_{r+\frac{1}{q}-1}^{(2)} \\
+H_{-\frac{1}{q}} H_{r+\frac{1}{q}-1}^{(2)}+\sum_{s=1}^{r-1} \frac{H_{s+\frac{1}{q}-1}^{(2)}}{s}
\end{array}\right]
$$


and since, from

$$
\sum_{n=1}^{\infty} \frac{h_{n}^{(2)}}{n\left(\begin{array}{c}
n+k \\
k
\end{array}\right)}=\sum_{n=1}^{\infty} \frac{H_{n-\frac{1}{q}}^{(2)}-H_{-\frac{1}{q}}^{(2)}}{n\left(\begin{array}{c}
n+k \\
k
\end{array}\right)}=\sum_{n=1}^{\infty} \frac{H_{n-\frac{1}{q}}^{(2)}}{n\left(\begin{array}{c}
n+k \\
k
\end{array}\right)}-\frac{H_{-\frac{1}{q}}^{(2)}}{k}
$$

then

$$
\begin{aligned}
& \sum_{n=1}^{\infty} \frac{H_{n-\frac{1}{q}}^{(2)}}{n\left(\begin{array}{c}
n+k \\
k
\end{array}\right)} \\
& =\frac{H_{-\frac{1}{q}}^{(2)}}{k}-H_{-\frac{1}{q}} H_{\frac{1}{q}-1}^{(2)}-H_{-\frac{1}{q}-1}\left(\zeta(2)-H_{-\frac{1}{q}}^{(2)}\right) \\
& +\sum_{r=1}^{k}(-1)^{r+1}\left(\begin{array}{c}
k \\
r
\end{array}\right)\left[\begin{array}{c}
H_{r+\frac{1}{q}-1}\left(\zeta(2)-H_{-\frac{1}{q}}^{(2)}\right)+H_{r+\frac{1}{q}-1}^{(2)}\left(H_{-\frac{1}{q}}-H_{r-1}\right) \\
+\sum_{s=1}^{r-1} \frac{H_{s+\frac{1}{q}-1}}{s}
\end{array}\right]
\end{aligned}
$$

hence the identity (2.1) follows.

The two special cases of $q= \pm 1$ follows in the next corollary.

Corollary 1. Under the assumptions of Theorem 1, from (2.1) let $q=1$, then

$$
\sum_{n=1}^{\infty} \frac{H_{n-1}^{(2)}}{n\left(\begin{array}{c}
n+k \\
k
\end{array}\right)}=\frac{\zeta(2)}{k^{2}}+\sum_{r=1}^{k}(-1)^{r}\left(\begin{array}{c}
k \\
r
\end{array}\right)\left(\frac{H_{r}}{r^{2}}+\sum_{j=1}^{r-1} \frac{H_{j}}{j^{2}}\right)
$$

and for $q=-1$,

$$
\begin{aligned}
\sum_{n=1}^{\infty} \frac{H_{n+1}^{(2)}}{n\left(\begin{array}{c}
n+k \\
k
\end{array}\right)=} & (1-k) \zeta(3)+\left(k H_{k-1}-k-H_{k-1}\right) \zeta(2)+3 k \\
& +\sum_{r=2}^{k}(-1)^{r+1}\left(\begin{array}{c}
k \\
r
\end{array}\right) \frac{H_{r}}{(r-1)^{2}}+\sum_{r=1}^{k}(-1)^{r}\left(\begin{array}{c}
k \\
r
\end{array}\right) \sum_{j=1}^{r-1} \frac{H_{j}}{j^{2}} \\
& -2 k H_{k-1}+\frac{k}{2}\left(H_{k-1}^{(2)}+H_{k-1}^{2}\right) .
\end{aligned}
$$

Proof. Consider $q=1$, then

$$
\sum_{n=1}^{\infty} \frac{H_{n-1}^{(2)}}{n\left(\begin{array}{c}
n+k \\
k
\end{array}\right)}=\sum_{n=1}^{\infty} \frac{H_{n}^{(2)}}{n\left(\begin{array}{c}
n+k \\
k
\end{array}\right)}-\sum_{n=1}^{\infty} \frac{1}{n^{3}\left(\begin{array}{c}
n+k \\
k
\end{array}\right)}
$$


The first sum on the right hand side of (2.6) can be replaced by (1.1), similarly

$$
\begin{aligned}
\sum_{n=1}^{\infty} \frac{1}{n^{3}\left(\begin{array}{c}
n+k \\
k
\end{array}\right)} & =\zeta(3)-H_{k} \zeta(2)+\sum_{r=1}^{k}(-1)^{r+1}\left(\begin{array}{c}
k \\
r
\end{array}\right) \frac{H_{r}}{r^{2}} \\
& =\frac{1}{k+1}{ }_{4} F_{3}\left[\begin{array}{c|c}
1,1,1,1 & 1 \\
2,2,2+k & 1
\end{array}\right]
\end{aligned}
$$

and substituting into (2.6) we obtain (2.4). For $q=-1$

$$
\begin{aligned}
\sum_{n=1}^{\infty} \frac{H_{n+1}^{(2)}}{n\left(\begin{array}{c}
n+k \\
k
\end{array}\right)=} & \sum_{n=1}^{\infty} \frac{H_{n}^{(2)}}{n\left(\begin{array}{c}
n+k \\
k
\end{array}\right)}+\sum_{n=1}^{\infty} \frac{1}{n(n+1)^{2}\left(\begin{array}{c}
n+k \\
k
\end{array}\right)} \\
= & \zeta(3)-H_{k-1} \zeta(2)+\sum_{r=1}^{k}(-1)^{r}\left(\begin{array}{c}
k \\
r
\end{array}\right) \sum_{j=1}^{r-1} \frac{H_{j}}{j^{2}} \\
& +\sum_{n=1}^{\infty} \frac{1}{n(n+1)^{2}\left(\begin{array}{c}
n+k \\
k
\end{array}\right) .}
\end{aligned}
$$

If we use the same method as in Theorem 1, by partial fraction expansion we are able to evaluate

$$
\begin{aligned}
\sum_{n=1}^{\infty} \frac{1}{n(n+1)^{2}\left(\begin{array}{c}
n+k \\
k
\end{array}\right)=} & -k \zeta(3)+k\left(H_{k-1}-1\right) \zeta(2)-2 k H_{k-1} \\
& +3 k+\frac{k}{2}\left(H_{k-1}^{(2)}+H_{k-1}^{2}\right) \\
& +\sum_{r=2}^{k}(-1)^{r+1}\left(\begin{array}{c}
k \\
r
\end{array}\right) \frac{H_{r}}{(r-1)^{2}},
\end{aligned}
$$

we can also evaluate

(2.8) $\sum_{n=1}^{\infty} \frac{1}{n(n+1)^{2}\left(\begin{array}{c}n+k \\ k\end{array}\right)}=2-{ }_{4} F_{3}\left[\begin{array}{c}1,1,1,1 \\ 2,2,1+k\end{array} \mid 1\right]+k H_{k}^{(2)}-k \zeta(2)$

then from $(2.7)$

$$
\begin{aligned}
\sum_{r=2}^{k}(-1)^{r}\left(\begin{array}{c}
k \\
r
\end{array}\right) \frac{H_{r}}{(r-1)^{2}}= & -k \zeta(3)+k H_{k-1} \zeta(2)-(2 k+1) H_{k} \\
& +3 k+\frac{k}{2}\left(H_{k}^{2}-H_{k}^{(2)}\right)+{ }_{4} F_{3}\left[\begin{array}{c}
1,1,1,1 \\
2,2,1+k
\end{array} \mid 1\right] .
\end{aligned}
$$


From (1.1) and (2.9), (2.5) follows. Rewriting the last equality we are able to write the one parameter hypergeometric identity

$$
{ }_{4} F_{3}\left[\begin{array}{c|c}
1,1,1,1 & 1 \\
2,2,1+k & 1
\end{array}\right]=k \zeta(3)-k H_{k-1} \zeta(2)-\sum_{r=2}^{k} \frac{(-1)^{r+1} r\left(\begin{array}{c}
k \\
r
\end{array}\right) H_{r-1}}{(r-1)^{2}} .
$$

From (2.8), after some manipulations and simplification we can also obtain some new identities, which will be useful in the following work:

$$
\begin{aligned}
& \sum_{r=2}^{k}(-1)^{r}\left(\begin{array}{c}
k \\
r
\end{array}\right) \frac{H_{r-1}}{r-1}=k H_{k}^{(2)}-H_{k}, \\
& \sum_{r=1}^{k}(-1)^{r}\left(\begin{array}{c}
k \\
r
\end{array}\right) H_{r+p}=H_{p}+\frac{1}{k\left(\begin{array}{c}
p+k \\
k
\end{array}\right)}, \\
& \sum_{r=1}^{k}(-1)^{r}\left(\begin{array}{c}
k \\
r
\end{array}\right) H_{r-1}^{(2)}=\frac{1}{2}\left(H_{k}^{(2)}+H_{k}^{2}\right)-\frac{H_{k}}{k} .
\end{aligned}
$$

Identity (2.10) holds, in general, for $k \in \mathbb{R} \backslash\{-1,-2,-3, \ldots\}$. If $k=\frac{1}{2}$ and using (1.8), then

$$
{ }_{4} F_{3}\left[\begin{array}{c|c}
1,1,1,1 & 1 \\
2,2,1+\frac{1}{2} & 1
\end{array}\right]=3 \ln 2 \zeta(2)-\frac{7}{4} \zeta(3) .
$$

Example 1. From Theorem 1, let $q=4, k=4$

$$
\sum_{n=1}^{\infty} \frac{H_{n-\frac{1}{4}}^{(2)}}{n\left(\begin{array}{c}
n+4 \\
4
\end{array}\right)}=\frac{45808}{38025}-\frac{3706}{195} G-\frac{1662976 \ln 2}{38025}+\frac{831488 \pi}{114075}+\frac{3771}{260} \zeta(2)
$$

for $q=-4, k=4$

$$
\sum_{n=1}^{\infty} \frac{H_{n+\frac{1}{4}}^{(2)}}{n\left(\begin{array}{c}
n+4 \\
4
\end{array}\right)}=152-\frac{140}{3} G-\frac{128 \ln 2}{3}-\frac{64 \pi}{9}-\frac{69}{2} \zeta(2),
$$

where $G=.915965 \cdots$ is the Catalan constant.

Remark 1. From Theorem 1 the case $q= \pm \frac{1}{m}, m \geq 1$ follows much the same procedure as in Theorem 1. Some specific cases are:

$$
\sum_{n=6}^{\infty} \frac{H_{n-6}^{(2)}}{n\left(\begin{array}{c}
n+3 \\
3
\end{array}\right)}=\sum_{n=1}^{\infty} \frac{H_{n}^{(2)}}{(n+6)\left(\begin{array}{c}
n+6+3 \\
3
\end{array}\right)}=\frac{1}{68} \zeta(2)-\frac{37073}{7902720}
$$


and

$$
\sum_{n=1}^{\infty} \frac{H_{n+6}^{(2)}}{n\left(\begin{array}{c}
n+3 \\
3
\end{array}\right)}=\frac{6401}{12000}-\frac{1}{60} \zeta(2) .
$$

Now we consider the following finite version of Theorem 1.

Theorem 2. Let $k, p \in \mathbb{N}$ and for real number $q>1$ and $q<-1$. Then we have

$$
\begin{aligned}
& \sum_{n=1}^{p} \frac{H_{n-\frac{1}{q}}^{(2)}}{n\left(\begin{array}{c}
n+k \\
k
\end{array}\right)} \\
= & q^{2} H_{p}+H_{-\frac{1}{q}}^{(2)}\left[\frac{1}{k}+H_{\frac{1}{q}-1}\right]-H_{p-\frac{1}{q}}^{(2)}\left(\frac{1}{k\left(\begin{array}{c}
p+k \\
k
\end{array}\right)}+H_{\frac{1}{q}-1}\right) \\
& -H_{\frac{1}{q}-1}^{(2)}\left[H_{-\frac{1}{q}}-H_{p-\frac{1}{q}}\right] \\
+ & \sum_{r=1}^{k}(-1)^{r+1}\left(\begin{array}{c}
k \\
r
\end{array}\right)\left[\begin{array}{c}
\left(H_{-\frac{1}{q}}-H_{p-\frac{1}{q}}-H_{r-1}\right) H_{r+\frac{1}{q}-1}^{(2)} \\
-\left(H_{-\frac{1}{q}}^{2}-H_{p-\frac{1}{q}}^{(2)}\right) H_{r+\frac{1}{q}-1} \\
+\sum_{m=1}^{r-1}\left(\left(\frac{q}{q m+1}\right)^{2} H_{m+p}+\frac{H_{m+\frac{1}{q}-1}^{(2)}}{m}\right)
\end{array}\right] .
\end{aligned}
$$

Proof. To prove (2.12) we may write

$$
\sum_{n=1}^{p} \frac{h_{n}^{(2)}}{n\left(\begin{array}{c}
n+k \\
k
\end{array}\right)}=\sum_{n=1}^{p} \frac{k ! h_{n}^{(2)}}{n} \sum_{r=1}^{k}\left(\frac{A_{r}}{n+r}\right),
$$

where $A_{r}$ is given by $(2.3)$, and by a rearrangement of sums

$$
\begin{aligned}
& \sum_{n=1}^{p} \frac{k ! h_{n}^{(2)}}{n} \sum_{r=1}^{k}\left(\frac{A_{r}}{n+r}\right) \\
= & \sum_{r=1}^{k}(-1)^{r+1} r\left(\begin{array}{c}
k \\
r
\end{array}\right) \sum_{j=1}^{p} \sum_{n=j}^{p}\left[\frac{1}{n(n+r)}\left(\frac{q}{q j-1}\right)^{2}\right] \\
= & \sum_{r=1}^{k}(-1)^{r+1} r\left(\begin{array}{c}
k \\
r
\end{array}\right) \sum_{j=1}^{p} \frac{1}{r}\left(\frac{q}{q j-1}\right)^{2}\left[\begin{array}{c}
\psi(r+j)-\psi(j) \\
-(\psi(p+1+j)-\psi(p+1))
\end{array}\right]
\end{aligned}
$$




$$
=\sum_{r=1}^{k}(-1)^{r+1}\left(\begin{array}{c}
k \\
r
\end{array}\right) \sum_{j=1}^{p}\left(\frac{q}{q j-1}\right)^{2}\left[\begin{array}{c}
\sum_{m=0}^{r-1} \frac{1}{m+j} \\
-\sum_{m=0}^{r-1} \frac{1}{m+p+1}
\end{array}\right] .
$$

From (1.3), (1.5) and (1.6)

$$
\begin{gathered}
\sum_{n=1}^{p} \frac{h_{n}^{(2)}}{n\left(\begin{array}{c}
n+k \\
k
\end{array}\right)} \\
=\sum_{r=1}^{k}(-1)^{r+1}\left(\begin{array}{c}
k \\
r
\end{array}\right) \sum_{m=0}^{r-1}\left[\begin{array}{c}
\frac{1}{p+m+1}\left(\psi^{\prime}\left(p-\frac{1}{q}+1\right)-\psi^{\prime}\left(1-\frac{1}{q}\right)\right) \\
+\frac{q}{q m+1}\left(\psi^{\prime}\left(1-\frac{1}{q}\right)-\psi^{\prime}\left(p+1-\frac{1}{q}\right)\right) \\
\left(\frac{q}{q m+1}\right)^{2}\left[\begin{array}{c}
\psi(m+p+1)-\psi(m+1) \\
\psi\left(1-\frac{1}{q}\right)-\psi\left(p+1-\frac{1}{q}\right)
\end{array}\right]
\end{array}\right] .
\end{gathered}
$$

$$
\begin{aligned}
& \sum_{n=1}^{p} \frac{h_{n}^{(2)}}{n\left(\begin{array}{c}
n+k \\
k
\end{array}\right)} \\
& =\sum_{r=1}^{k}(-1)^{r+1}\left(\begin{array}{c}
k \\
r
\end{array}\right) \sum_{m=0}^{r-1}\left(\frac{q}{q m+1}\right)^{2}\left(H_{m+p}-H_{m}\right) \\
& +\sum_{r=1}^{k}(-1)^{r+1}\left(\begin{array}{c}
k \\
r
\end{array}\right)\left[\begin{array}{c}
\left(H_{p+r}-H_{p}\right)\left(H_{-\frac{1}{q}}^{(2)}-H_{p-\frac{1}{q}}^{(2)}\right) \\
+\left(H_{r+\frac{1}{q}-1}^{(2)}-H_{\frac{1}{q}-1}^{(2)}\right)\left(H_{-\frac{1}{q}}-H_{p-\frac{1}{q}}\right) \\
+\left(H_{r+\frac{1}{q}-1}-H_{\frac{1}{q}-1}\right)\left(H_{p-\frac{1}{q}}^{(2)}-H_{-\frac{1}{q}}^{(2)}\right)
\end{array}\right] \\
& =q^{2} H_{p}+\sum_{r=1}^{k}(-1)^{r+1}\left(\begin{array}{c}
k \\
r
\end{array}\right) \sum_{m=1}^{r-1}\left(\frac{q}{q m+1}\right)^{2}\left(H_{m+p}-H_{m}\right) \\
& +\frac{1}{k\left(\begin{array}{c}
p+k \\
k
\end{array}\right)}\left(H_{-\frac{1}{q}}^{(2)}-H_{p-\frac{1}{q}}^{(2)}\right) \\
& -H_{\frac{1}{q}-1}^{(2)}\left(H_{-\frac{1}{q}}-H_{p-\frac{1}{q}}\right)-H_{\frac{1}{q}-1}\left(H_{p-\frac{1}{q}}^{(2)}-H_{-\frac{1}{q}}^{(2)}\right) \\
& +\sum_{r=1}^{k}(-1)^{r+1}\left(\begin{array}{c}
k \\
r
\end{array}\right)\left[H_{r+\frac{1}{q}-1}^{(2)}\left(H_{-\frac{1}{q}}-H_{p-\frac{1}{q}}\right)-H_{r+\frac{1}{q}-1}\left(H_{-\frac{1}{q}}^{(2)}-H_{p-\frac{1}{q}}^{(2)}\right)\right] \text {. }
\end{aligned}
$$


Since from

$$
\begin{aligned}
\sum_{n=1}^{p} \frac{h_{n}^{(2)}}{n\left(\begin{array}{c}
n+k \\
k
\end{array}\right)} & =\sum_{n=1}^{p} \frac{H_{n-\frac{1}{q}}^{(2)}-H_{-\frac{1}{q}}^{(2)}}{n\left(\begin{array}{c}
n+k \\
k
\end{array}\right)} \\
& =\sum_{n=1}^{p} \frac{H_{n-\frac{1}{q}}^{(2)}}{n\left(\begin{array}{c}
n+k \\
k
\end{array}\right)}-\frac{H_{-\frac{1}{q}}^{(2)}}{k}\left[1-\frac{1}{\left(\begin{array}{c}
p+k \\
k
\end{array}\right)}\right]
\end{aligned}
$$

substituting into (2.13) and after simplification (2.12) follows.

The two special cases of $q= \pm 1$ follows in the next corollary and we will also give two remarkable identities for hypergeometric functions containing two parameters.

Corollary 2. Under the assumptions of Theorem 2, from (2.12) let $q=-1$, then

$$
\begin{aligned}
\sum_{n=1}^{p} \frac{H_{n+1}^{(2)}}{n\left(\begin{array}{c}
n+k \\
k
\end{array}\right)=} & H_{p}^{(3)}-H_{p}^{(2)}\left(H_{k-1}+\frac{1}{k\left(\begin{array}{c}
k+p \\
k
\end{array}\right)}\right) \\
& +\frac{H_{p}}{2}\left(H_{k}^{(2)}+H_{p}^{2}-\frac{2 H_{k}}{k}\right) \\
& +\sum_{r=2}^{k}(-1)^{r+1}\left(\begin{array}{c}
k \\
r
\end{array}\right) \frac{H_{r-1}-H_{p+r}+H_{p+1}}{(r-1)^{2}} \\
& +\sum_{r=1}^{k}(-1)^{r+1}\left(\begin{array}{c}
k \\
r
\end{array}\right) \sum_{j=1}^{r-1} \frac{H_{p+j}-H_{j}}{j^{2}} \\
& +\frac{2 p+1}{p+1}+H_{k}-k\left(H_{p+1}^{(2)}+H_{p+1}^{(3)}-H_{p+1}^{(2)} H_{k-1}\right)
\end{aligned}
$$

and for $q=1$

$(2.15)$

$$
\begin{aligned}
\sum_{n=1}^{p} \frac{H_{n-1}^{(2)}}{n\left(\begin{array}{c}
n+k \\
k
\end{array}\right)=} & \frac{H_{p}^{(2)}}{k}\left(1-\frac{1}{\left(\begin{array}{c}
k+p \\
k
\end{array}\right)}\right)-\frac{H_{k} H_{p}}{k} \\
& +\sum_{r=1}^{k}(-1)^{r}\left(\begin{array}{c}
k \\
r
\end{array}\right)\left[\frac{H_{r}-H_{p+r}}{r^{2}}-\sum_{j=1}^{r-1} \frac{H_{j+p}-H_{j}}{j^{2}}\right]
\end{aligned}
$$


Proof. First let us consider the case $q=-1$. Let

$$
\begin{aligned}
& \sum_{n=1}^{p} \frac{1}{n(n+1)^{2}\left(\begin{array}{c}
n+k \\
k
\end{array}\right)} \\
& =\sum_{n=1}^{\infty} \frac{1}{n(n+1)^{2}\left(\begin{array}{c}
n+k \\
k
\end{array}\right)}-\sum_{n=p+1}^{\infty} \frac{1}{n(n+1)^{2}\left(\begin{array}{c}
n+k \\
k
\end{array}\right)} \\
& =\frac{2 k+1}{k}-\frac{1}{k\left(\begin{array}{c}
p+k \\
k
\end{array}\right)}-k \zeta(2)+k H_{k-1}^{(2)} \\
& -\frac{1}{(p+2)\left(\begin{array}{c}
p+k+1 \\
k
\end{array}\right)}{ }_{3} F_{2}\left[\begin{array}{c|c}
1, p+2, p+2 \\
p+3, p+k+2
\end{array}\right] \\
& +\frac{1}{(p+2)^{2}\left(\begin{array}{c}
p+k+1 \\
k
\end{array}\right)}{ }_{4} F_{3}\left[\begin{array}{c|c}
1, p+2, p+2, p+2 \\
p+3, p+3, p+k+2
\end{array} \mid 1\right] \\
& -{ }_{4} F_{3}\left[\begin{array}{c|c}
1,1,1,1 & 1 \\
2,2,1+k & 1
\end{array}\right] \text {. }
\end{aligned}
$$

Now, by partial fraction decomposition we have

$$
\begin{aligned}
\sum_{n=1}^{p} \frac{1}{n(n+1)^{2}\left(\begin{array}{c}
n+k \\
k
\end{array}\right)=} & \frac{2 p+1}{p+1}-k\left(H_{p+1}^{(2)}+H_{p+1}^{(3)}-H_{p+1}^{(2)} H_{k-1}\right) \\
& +H_{k}-\frac{k(k-1)}{2} H_{p+1} F_{3}\left[\begin{array}{c}
1,1,2,2-k \\
2,2,3
\end{array} \mid 1\right] \\
& +\sum_{r=2}^{k}(-1)^{r+1}\left(\begin{array}{c}
k \\
r
\end{array}\right)\left(\frac{H_{r-1}-H_{p+r}}{(r-1)^{2}}\right)
\end{aligned}
$$

and from (2.7) and (2.10) we know that

$$
\begin{aligned}
& \sum_{n=1}^{\infty} \frac{1}{n(n+1)^{2}\left(\begin{array}{c}
n+k \\
k
\end{array}\right)} \\
= & \frac{2 p+1}{p+1}+H_{k}-k\left(H_{p+1}^{(2)}+H_{p+1}^{(3)}-H_{p+1}^{(2)} H_{k-1}\right) \\
& +\sum_{r=2}^{k}(-1)^{r+1}\left(\begin{array}{c}
k \\
r
\end{array}\right) \frac{H_{p+1}}{(r-1)^{2}}+\sum_{r=2}^{k}(-1)^{r+1}\left(\begin{array}{c}
k \\
r
\end{array}\right)\left(\frac{H_{r-1}-H_{p+r}}{(r-1)^{2}}\right) .
\end{aligned}
$$


The last step is to write

$$
\sum_{n=1}^{p} \frac{H_{n+1}^{(2)}}{n\left(\begin{array}{c}
n+k \\
k
\end{array}\right)}=\sum_{n=1}^{p} \frac{H_{n}^{(2)}}{n\left(\begin{array}{c}
n+k \\
k
\end{array}\right)}+\sum_{n=1}^{p} \frac{1}{n(n+1)^{2}\left(\begin{array}{c}
n+k \\
k
\end{array}\right)}
$$

substituting the known result (1.2), and (2.17) into (2.18) we obtain, upon using (2.11), the identity (2.14). From (2.16), (2.17) and (2.7) we obtain the new, two parameter identity

$$
\begin{aligned}
& \frac{{ }_{3} F_{2}\left[\begin{array}{c}
1, p+1, p+2 \\
p+3, p+k+2
\end{array}\right.}{(p+2)\left(\begin{array}{c}
p+k+1 \\
k
\end{array}\right)} \\
= & \zeta(2)-H_{p+1}^{(2)}+H_{p+1} H_{k-1}+\sum_{r=2}^{k}(-1)^{r+1} r\left(\begin{array}{c}
k \\
r
\end{array}\right) \frac{H_{p+r}}{r-1}
\end{aligned}
$$

and

$$
\begin{aligned}
& (p+1){ }_{3} F_{2}\left[\begin{array}{c}
1, p+2, p+2 \\
p+3, p+k+2
\end{array} \mid 1\right]+{ }_{3} F_{2}\left[\begin{array}{c|c}
1, p+1, p+2 \\
p+3, p+k+2
\end{array} \mid 1\right] \\
= & \frac{(p+2)(p+k+1)}{k} .
\end{aligned}
$$

For $q=1$, let

$$
\sum_{n=1}^{p} \frac{H_{n-1}^{(2)}}{n\left(\begin{array}{c}
n+k \\
k
\end{array}\right)}=\sum_{n=1}^{p}\left[\frac{H_{n}^{(2)}}{n\left(\begin{array}{c}
n+k \\
k
\end{array}\right)}-\frac{1}{n^{3}\left(\begin{array}{c}
n+k \\
k
\end{array}\right)}\right]
$$

and by partial fraction expansion

$$
\begin{aligned}
\sum_{n=1}^{p} \frac{1}{n^{3}\left(\begin{array}{c}
n+k \\
k
\end{array}\right)=} & H_{p}^{(3)}-H_{p}^{(2)} H_{k}+\frac{H_{p}}{2}\left(H_{k}^{(2)}+H_{k}^{2}\right) \\
& +\sum_{r=1}^{k}(-1)^{r+1}\left(\begin{array}{c}
k \\
r
\end{array}\right) \frac{\left(H_{r}-H_{p+r}\right)}{r^{2}} .
\end{aligned}
$$

Similarly

$$
\sum_{n=1}^{p} \frac{1}{n^{3}\left(\begin{array}{c}
n+k \\
k
\end{array}\right)}
$$




$$
\begin{aligned}
& =\sum_{n=1}^{\infty} \frac{1}{n^{3}\left(\begin{array}{c}
n+k \\
k
\end{array}\right)}-\sum_{n=p+1}^{\infty} \frac{1}{n^{3}\left(\begin{array}{c}
n+k \\
k
\end{array}\right)}
\end{aligned}
$$

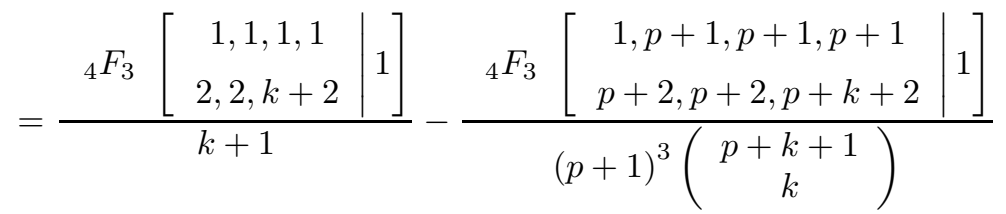

and from $(2.21),(2.22)$ and (2.11) we obtain the new two parameter hypergeometric identity

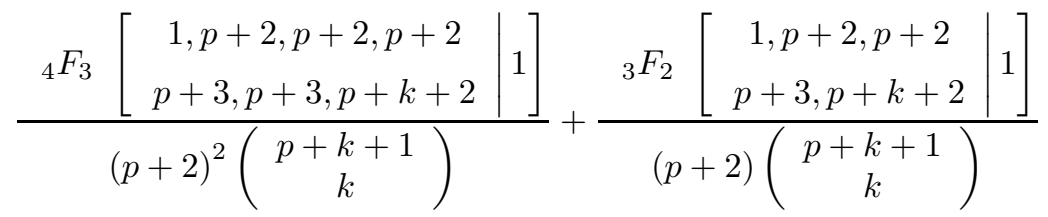

$$
\begin{aligned}
& =k \zeta(3)+k\left(1-H_{k-1}\right)\left(\zeta(2)-H_{p+1}^{(2)}\right)-k H_{p+1}^{(3)} \\
& +\sum_{r=1}^{k}(-1)^{r+1}\left(\begin{array}{c}
k \\
r
\end{array}\right)\left(\frac{H_{p+1}-H_{p+r}}{(r-1)^{2}}\right) .
\end{aligned}
$$

Using the identities (1.2) and (2.21) into (2.20) and simplifying we obtain the required identity $(2.15)$. From (2.19) and (2.23) we can also explicitly derive an identity for ${ }_{4} F_{3}\left[\begin{array}{c|c}1, p+2, p+2, p+2 \\ p+3, p+3, p+k+2\end{array} \mid 1\right]$.

Identities (2.19) and (2.23) hold, in general, for $p \in \mathbb{R} \backslash\{-1,-2,-3, \ldots$,$\} .$ If $p=\frac{3}{4}, k=5$ and using (1.8), then

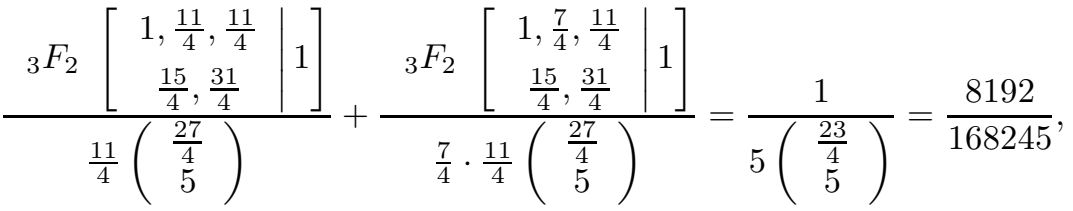

and

$$
\begin{gathered}
\frac{{ }_{4} F_{3}\left[\begin{array}{c}
1, \frac{11}{4}, \frac{11}{4}, \frac{11}{4} \\
\frac{15}{4}, \frac{15}{4}, \frac{31}{4}
\end{array} \mid 1\right]}{\left(\frac{11}{4}\right)^{2}\left(\begin{array}{c}
\frac{27}{4} \\
5
\end{array}\right)}+\frac{{ }_{3} F_{2}\left[\begin{array}{c}
1, \frac{11}{4}, \frac{11}{4} \\
\frac{15}{4}, \frac{31}{4}
\end{array}\right]}{\frac{11}{4}\left(\begin{array}{c}
\frac{27}{4} \\
5
\end{array}\right)} \\
=140 \zeta(3)-5 \pi^{3}-\frac{65}{2} \zeta(2)+\frac{130}{3} G+\frac{24712405}{44517627} .
\end{gathered}
$$


Example 2. Some examples follow. Using the identities (2.11), (2.12), (2.14) and (2.15), then

$$
\begin{aligned}
\sum_{n=1}^{p} \frac{H_{n+\frac{1}{4}}^{(2)}}{n\left(\begin{array}{c}
n+2 \\
2
\end{array}\right)}= & 152-\frac{140}{3} G+\frac{16(7 p+8)}{9 p(p+1)}-\frac{64}{9} \pi-\frac{175}{6} \zeta(2)+128 H_{p-1} \\
& -\frac{128}{9} H_{p+\frac{1}{4}}-\frac{128}{3} \ln (2)-H_{p+\frac{1}{4}}^{(2)}\left(\frac{16}{3}+\frac{1}{(p+1)(p+2)}\right), \\
\sum_{n=1}^{p} \frac{H_{n-\frac{1}{4}}^{(2)}}{n\left(\begin{array}{c}
n+2 \\
2
\end{array}\right)}= & \frac{16}{25}-\frac{108}{5} G+\frac{16(23 p+24)}{25 p(p+1)}+\frac{192}{25} \pi+\frac{27}{2} \zeta(2)+\frac{384}{25} H_{p-1} \\
\sum_{n=1}^{p} \frac{H_{n+1}^{(2)}}{n\left(\begin{array}{c}
n+2 \\
2
\end{array}\right)}= & \frac{7 p^{3}+19 p^{2}+17 p+1}{2(p+1)^{3}}-\frac{H_{p}}{p+2}-\frac{H_{p}^{(2)}\left(p^{2}+3 p+3\right)}{(p+1)(p+2)}-H_{p}^{(3)} .
\end{aligned}
$$

Also,

$$
\begin{aligned}
& \sum_{n=1}^{p} \frac{H_{n+1}^{(2)}}{n(n+1)}=\frac{3 p+2}{p+1}-\frac{p+2}{p+1} H_{p+1}^{(2)}, \\
& \sum_{n=1}^{p} \frac{H_{n-1}^{(2)}}{n(n+1)}=\frac{p}{p+1}\left(H_{p}^{(2)}-1\right) .
\end{aligned}
$$

Remark 2. Using similar principles as discussed above, it is possible to express the more general sums, $\sum_{n=1}^{\infty} \frac{H_{n-\frac{1}{q}}^{(p)}}{n^{r}\left(\begin{array}{c}n+k \\ k\end{array}\right)^{m}}$, in closed form. This will be done in a forthcoming paper.

\section{References}

[1] J. Choi, Finite summation formulas involving binomial coefficients, harmonic numbers and generalized harmonic numbers, J. Ineq. Appl. 49 (2013), 11 p.

[2] — Certain summation formulas involving harmonic numbers and generalized harmonic numbers, Appl. Math. Comput. 218 (2011), no. 3, 734-740.

[3] J. Choi and D. Cvijović, Values of the polygamma functions at rational arguments, J. Phys. A: Math. Theor. 40 (2007), no. 50, 15019-15028; Corrigendum, ibidem 43 (2010), no. 23, 239801, 1p.

[4] J. Choi and H. M. Srivastava, Some summation formulas involving harmonic numbers and generalized harmonic numbers, Math. Comp. Modelling 54 (2011), no. 9-10, 22202234.

[5] W. Chu, Summation formulae involving harmonic numbers, Filomat. 26 (2012), no. 1, $143-152$.

[6] - Infinite series identities on harmonic numbers, Results Math. 61 (2012), no. 3-4, 209-221. 
[7] A. Dil and V. Kurt, Polynomials related to harmonic numbers and evaluation of harmonic number series II, Appl. Anal. Discrete Math. 5 (2011), no. 2, 212-229.

[8] H.-T. Jin and L. H. Sun, On Spieß's conjecture on harmonic numbers, Discrete Appl. Math. 161 (2013), no. 13-14, 2038-2041.

[9] K. Kölbig, The polygamma function $\psi(x)$ for $x=1 / 4$ and $x=3 / 4$, J. Comput. Appl. Math. 75 (1996), no. 1, 43-46.

[10] H. Liu and W. Wang, Harmonic number identities via hypergeometric series and Bell polynomials, Integral Transforms Spec. Funct. 23 (2012), no. 1, 49-68.

[11] E. Munarini, Riordan matrices and sums of harmonic numbers, Appl. Anal. Discrete Math. 5 (2011), no. 2, 176-200.

[12] A. Sofo, Computational Techniques for the Summation of Series, Kluwer Academic/ Plenum Publishers, New York, 2003.

[13] Sums of derivatives of binomial coefficients, Adv. in Appl. Math. 42 (2009), no. $1,123-134$.

[14] _ Harmonic sums and integral representations, J. Appl. Anal. 16 (2010), no. 2, $265-277$.

[15] _ Harmonic number sums in higher powers, J. Math. Anal. 2 (2011), no. 2, 15-22.

[16] Summation formula involving harmonic numbers, Anal. Math. 37 (2011), no. $1,51-64$.

[17] , New classes of harmonic number identities, J. Integer Seq. 15 (2012), Article 12.7.4.

[18] - Finite number sums in higher order powers of harmonic numbers, Bull. Math. Anal. Appl. 5 (2013), no. 1, 71-79.

[19] _ Mixed binomial sum identities, Integral Transforms Spec. Funct. 24 (2013), no. $3,187-200$.

[20] A. Sofo and H. M. Srivastava, Identities for the harmonic numbers and binomial coefficients, Ramanujan J. 25 (2011), no. 1, 93-113.

[21] H. M. Srivastava and J. Choi, Series Associated with the Zeta and Related Functions, Kluwer Academic Publishers, London, 2001.

VICTORIA UNIVERSITY

P.O. Box 14428, Melbourne City

VIC 8001, Australia

E-mail address: anthony.sofo@vu.edu.au 